\title{
On Classical and Quantum Logical Entropy
}

\author{
David Ellerman \\ University of California at Riverside
}

April 17, 2016

\begin{abstract}
The notion of a partition on a set is mathematically dual to the notion of a subset of a set, so there is a logic of partitions dual to Boole's logic of subsets (Boolean logic is usually mis-specified as "propositional" logic). The notion of an element of a subset has as its dual the notion of a distinction of a partition (a pair of elements in different blocks). Boole developed finite logical probability as the normalized counting measure on elements of subsets so there is a dual concept of logical entropy which is the normalized counting measure on distinctions of partitions. Thus the logical notion of information is a measure of distinctions. Classical logical entropy naturally extends to the notion of quantum logical entropy which provides a more natural and informative alternative to the usual Von Neumann entropy in quantum information theory. The quantum logical entropy of a post-measurement density matrix has the simple interpretation as the probability that two independent measurements of the same state using the same observable will have different results. The main result of the paper is that the increase in quantum logical entropy due to a projective measurement of a pure state is the sum of the absolute squares of the off-diagonal entries ("coherences") of the pure state density matrix that are zeroed ("decohered") by the measurement, i.e., the measure of the distinctions ("decoherences") created by the measurement.
\end{abstract}

\section{Contents}

1 Duality of subsets and partitions 1

2 Classical subset logic and partition logic 2

3 Classical probability and classical logical entropy 3

4 Classical logical entropy of a density matrix 5

5 Quantum logical entropy and measurement 6

6 Concluding remarks $\quad 10$

\section{Duality of subsets and partitions}

Logical entropy is to the logic of partitions as logical probability is to the Boolean logic of subsets. Hence we will start with a brief review of the relationship between these two dual forms of logic.

Modern category theory shows that the concept of a subset dualizes to the concept of a quotient set, equivalence relation, or partition. F. William Lawvere called a subset or, in general, a subobject a "part" and then noted: "The dual notion (obtained by reversing the arrows) of 'part' is the notion 
of partition." [14, p. 85] That suggests that the Boolean logic of subsets (usually named after the special case of propositions as "propositional" logic) should have a dual logic of partitions ([6], [10]).

A partition $\pi=\left\{B_{1}, \ldots, B_{m}\right\}$ on $U$ is a set of subsets or "blocks" $B_{i}$ that are mutually disjoint and jointly exhaustive $\left(\cup_{i} B_{i}=U\right)$. In the duality between subset logic and partition logic, the dual to the notion of an "element" of a subset is the notion of a "distinction" of a partition, where $\left(u, u^{\prime}\right) \in U \times U$ is a distinction or dit of $\pi$ if the two elements are in different blocks. Let $\operatorname{dit}(\pi) \subseteq U \times U$ be the set of distinctions or ditset of $\pi$. Similarly an indistinction or indit of $\pi$ is a pair $\left(u, u^{\prime}\right) \in U \times U$ in the same block of $\pi$. Let indit $(\pi) \subseteq U \times U$ be the set of indistinctions or inditset of $\pi$. Then indit $(\pi)$ is the equivalence relation associated with $\pi$ and $\operatorname{dit}(\pi)=U \times U-\operatorname{indit}(\pi)$ is the complementary binary relation that might be called a partition relation or an apartness relation.

\section{Classical subset logic and partition logic}

The algebra associated with the subsets $S \subseteq U$ is, of course, the Boolean algebra $\wp(U)$ of subsets of $U$ with the partial order as the inclusion of elements. The corresponding algebra of partitions $\pi$ on $U$ is the partition algebra $\prod(U)$ defined as follows:

- the partial order $\sigma \preceq \pi$ of partitions $\sigma=\left\{C, C^{\prime}, \ldots\right\}$ and $\pi=\left\{B, B^{\prime}, \ldots\right\}$ holds when $\pi$ refines $\sigma$ in the sense that for every block $B \in \pi$ there is a block $C \in \sigma$ such that $B \subseteq C$, or, equivalently, using the element-distinction pairing, the partial order is the inclusion of distinctions: $\sigma \preceq \pi$ if and only if (iff) $\operatorname{dit}(\sigma) \subseteq \operatorname{dit}(\pi)$;

- the minimum or bottom partition is the indiscrete partition (or blob) $\mathbf{0}=\{U\}$ with one block consisting of all of $U$;

- the maximum or top partition is the discrete partition $\mathbf{1}=\left\{\left\{u_{j}\right\}\right\}_{j=1, \ldots, n}$ consisting of singleton blocks;

- the join $\pi \vee \sigma$ is the partition whose blocks are the non-empty intersections $B \cap C$ of blocks of $\pi$ and blocks of $\sigma$, or, equivalently, using the element-distinction pairing, $\operatorname{dit}(\pi \vee \sigma)=$ $\operatorname{dit}(\pi) \cup \operatorname{dit}(\sigma)$;

- the meet $\pi \wedge \sigma$ is the partition of equivalence classes for the equivalence relation generated by: $u_{j} \sim u_{j^{\prime}}$ if $u_{j} \in B \in \pi, u_{j^{\prime}} \in C \in \sigma$, and $B \cap C \neq \emptyset$; and

- $\sigma \Rightarrow \pi$ is the implication partition whose blocks are: (1) the singletons $\left\{u_{j}\right\}$ for $u_{j} \in B \in \pi$ if there is a $C \in \sigma$ such that $B \subseteq C$, or (2) just $B \in \pi$ if there is no $C \in \sigma$ with $B \subseteq C$, so that trivially: $\sigma \Rightarrow \pi=\mathbf{1}$ iff $\sigma \preceq \pi$. $^{1}$

Since the same operations can be defined for subsets and partitions, one can interpret a formula $\Phi(\pi, \sigma, \ldots)$ either way as a subset or a partition. Given either subsets on or partitions of $U$ substituted for the variables $\pi, \sigma, \ldots$, one can apply, respectively, subset or partition operations to evaluate the whole formula. Since $\Phi(\pi, \sigma, \ldots)$ is either a subset or a partition, the corresponding proposition is " $u$ is an element of $\Phi(\pi, \sigma, \ldots)$ " or " $\left(u, u^{\prime}\right)$ is a distinction of $\Phi(\pi, \sigma, \ldots) "$. And then the definitions of a valid formula are also parallel, namely, no matter what is substituted for the variables, the whole formula evaluates to the top of the algebra. In that case, the subset $\Phi(\pi, \sigma, \ldots)$ contains all elements of $U$, i.e., $\Phi(\pi, \sigma, \ldots)=U$, or the partition $\Phi(\pi, \sigma, \ldots)$ distinguishes all pairs $\left(u, u^{\prime}\right)$ for distinct elements of $U$, i.e., $\Phi(\pi, \sigma, \ldots)=\mathbf{1}$. The parallelism between the dual logics is summarized in the following figure.

\footnotetext{
${ }^{1}$ There is a general method to define operations on partitions corresponding to operations on subsets ([6], [10]) but the lattice operations of join and meet, and the implication operation are sufficient to define a partition algebra $\prod(U)$ parallel to the familiar powerset Boolean algebra $\wp(U)$.
} 


\begin{tabular}{|c|c|c|}
\hline & Subset Logic & Partition Logic \\
\hline 'Elements' & Elements u of a subset $\mathrm{S}$ & Distinctions $\left(\mathrm{u}, \mathrm{u}^{\prime}\right)$ of a partition $\pi$ \\
\hline All 'elements' & Universe set U (all elements) & Discrete partition 1 (all dits) \\
\hline No 'elements' & Empty set $\varnothing$ (no elements) & Indiscrete partition $\mathbf{0}$ (no dits) \\
\hline Duality & $\begin{array}{l}\text { Subsets are images } \mathrm{f}() \text { of } \\
\text { injections } \mathrm{f}: \mathrm{S} \rightarrow \mathrm{U}\end{array}$ & $\begin{array}{l}\text { Partitions are inverse-images } \\
\mathrm{f}^{-1}() \text { of surjections } \mathrm{f}: \mathrm{U} \rightarrow \mathrm{T}\end{array}$ \\
\hline Partial order & $\begin{array}{l}\text { Inclusion of elements } \\
\text { (inclusion order on subsets) }\end{array}$ & $\begin{array}{l}\text { Inclusion of distinctions (dits) } \\
\text { (refinement order on partitions) }\end{array}$ \\
\hline Formula variables & Subsets of $U$ & Partitions on $\mathrm{U}$ \\
\hline $\begin{array}{l}\text { Logical operations } \\
\cup, \cap, \Rightarrow, \ldots\end{array}$ & Operations on subsets & Operations on partitions \\
\hline $\begin{array}{l}\text { Propositional } \\
\text { interp. of } \Phi(\pi, \sigma, \ldots)\end{array}$ & $\begin{array}{l}\text { Subset } \Phi(\pi, \sigma, \ldots) \text { has element } \\
\text { u. }\end{array}$ & $\begin{array}{l}\text { Partition } \Phi(\pi, \sigma, \ldots) \text { has distinction } \\
\left(\mathrm{u}, \mathrm{u}^{\prime}\right) \text {. }\end{array}$ \\
\hline $\begin{array}{l}\text { Valid formula } \\
\Phi(\pi, \sigma, \ldots)\end{array}$ & $\begin{array}{l}\Phi(\pi, \sigma, \ldots)=U \text { for any subsets } \\
\pi, \sigma, \ldots \text { of any } U(|U| \geq 1) \text {, i.e., } \\
\text { contains all elements } u \text {. }\end{array}$ & $\begin{array}{l}\Phi(\pi, \sigma, \ldots)=\mathbf{1} \text { for any partitions } \\
\pi, \sigma, \ldots \text { on any } \mathrm{U}(|\mathrm{U}| \geq 2), \text { i.e., } \\
\text { makes all distinctions }\left(\mathrm{u}, \mathrm{u}^{\prime}\right) \text {. }\end{array}$ \\
\hline
\end{tabular}

Figure 1: Boolean subset logic and partition logic.

\section{Classical probability and classical logical entropy}

George Boole [3] extended his logic of subsets to classical finite probability theory where, in the equiprobable case, the probability of a subset $S$ (event) of a finite universe set (outcome set or sample space) $U=\left\{u_{1}, \ldots, u_{n}\right\}$ was the number of elements in $S$ over the total number of elements: $\operatorname{Pr}(S)=\frac{|S|}{|U|}=\sum_{u_{j} \in S} \frac{1}{|U|}$. Classical finite probability theory [13] also dealt with the case where the outcomes were assigned real point probabilities $p=\left\{p_{1}, \ldots, p_{n}\right\}$ (where $p_{j} \geq 0$ and $\sum_{j} p_{j}=1$ ) so rather than summing the equal probabilities $\frac{1}{|U|}$, the point probabilities of the elements were summed: $\operatorname{Pr}(S)=\sum_{u_{j} \in S} p_{j}=p(S)$-where the equiprobable formula is for $p_{j}=\frac{1}{|U|}$ for $j=1, \ldots, n$. The conditional probability of an event $T \subseteq U$ given an event $S$ is $\operatorname{Pr}(T \mid S)=\frac{p(T \cap S)}{p(S)}$. Given a real-valued random variable $f: U \rightarrow \mathbb{R}$ on the outcome set $U$, the possible values of $f$ are $f(U)=\left\{\phi_{1}, \ldots, \phi_{m}\right\}$ and the probability of getting a certain value given $S$ is: $\operatorname{Pr}\left(\phi_{i} \mid S\right)=\frac{p\left(f^{-1}\left(\phi_{i}\right) \cap S\right)}{p(S)}$.

Then we may mimic Boole's move going from the logic of subsets to the finite logical probabilities of subsets by starting with the logic of partitions and using the dual relation between elements and distinctions. The dual notion to probability turns out to be "information content" or "entropy" so we define the logical entropy of $\pi$, denoted $h(\pi)$, as the size of the $\operatorname{ditset} \operatorname{dit}(\pi) \subseteq U \times U$ normalized by the size of $U \times U$ :

$$
\begin{gathered}
h(\pi)=\frac{|\operatorname{dit}(\pi)|}{|U \times U|}=\sum_{\left(u_{j}, u_{k}\right) \in \operatorname{dit}(\pi)} \frac{1}{|U|} \frac{1}{|U|} \\
\text { Logical entropy of } \pi \text { (equiprobable case). }
\end{gathered}
$$

This definition corresponds to the equiprobable case $\operatorname{Pr}(S)=\frac{|S|}{|U|}$ of the normalized number of elements rather than normalized number of distinctions. The corresponding definition for the case 
of point probabilities $p=\left\{p_{1}, \ldots, p_{n}\right\}$ is to just add up the probabilities of getting a particular distinction:

$$
h_{p}(\pi)=\sum_{\left(u_{j}, u_{k}\right) \in \operatorname{dit}(\pi)} p_{j} p_{k}
$$

Logical entropy of $\pi$ with point probabilities $p$.

There is another way to express these logical entropies. The inditset of $\pi$ is indit $(\pi)=\cup_{i=1}^{m}\left(B_{i} \times B_{i}\right)$ so where $p\left(B_{i}\right)=\frac{\left|B_{i}\right|}{|U|}$ in the equiprobable case, we have:

$$
h(\pi)=\frac{|\operatorname{dit}(\pi)|}{|U \times U|}=\frac{|U \times U|-\sum_{i=1}^{m}\left|B_{i} \times B_{i}\right|}{|U \times U|}=1-\sum_{i=1}^{m}\left(\frac{\left|B_{i}\right|}{|U|}\right)^{2}=1-\sum_{i=1}^{m} p\left(B_{i}\right)^{2} .
$$

This suggest that in the case of point probabilities, we should take $p\left(B_{i}\right)=\sum_{u_{j} \in B_{i}} p_{j}$ and have $h_{p}(\pi)=1-\sum_{i=1}^{m} p\left(B_{i}\right)^{2}$. This is confirmed with a little calculation using that definition of $p\left(B_{i}\right)$ :

$$
1=\left[p\left(B_{1}\right)+\ldots+p\left(B_{m}\right)\right]\left[p\left(B_{1}\right)+\ldots+p\left(B_{m}\right)\right]=\sum_{i=1}^{m} p\left(B_{i}\right)^{2}+\sum_{i \neq i^{\prime}} p\left(B_{i}\right) p\left(B_{i^{\prime}}\right)
$$

so that:

$$
1-\sum_{i=1}^{m} p\left(B_{i}\right)^{2}=\sum_{i \neq i^{\prime}} p\left(B_{i}\right) p\left(B_{i^{\prime}}\right) .^{2}
$$

Moreover, we have:

$$
\sum_{i=1}^{m} p\left(B_{i}\right)^{2}=\sum_{i}\left(\sum_{u_{j} \in B_{i}} p_{j}\right)^{2}=\sum_{i} \sum_{\left(u_{j}, u_{k}\right) \in B_{i} \times B_{i}} p_{j} p_{k}=\sum_{\left(u_{j}, u_{k}\right) \in \operatorname{indit}(\pi)} p_{j} p_{k}
$$

so that:

$$
1-\sum_{i=1}^{m} p\left(B_{i}\right)^{2}=\sum_{i \neq i^{\prime}} p\left(B_{i}\right) p\left(B_{i^{\prime}}\right)=1-\sum_{\left(u_{j}, u_{k}\right) \in \operatorname{indit}(\pi)} p_{j} p_{k}=\sum_{\left(u_{j}, u_{k}\right) \in \operatorname{dit}(\pi)} p_{j} p_{k}
$$

since:

$$
\begin{aligned}
1= & \left(p_{1}+\ldots+p_{n}\right)\left(p_{1}+\ldots+p_{n}\right)=\sum_{\left(u_{j}, u_{k}\right) \in U \times U} p_{j} p_{k} \\
& =\sum_{\left(u_{j}, u_{k}\right) \in \operatorname{indit}(\pi)} p_{j} p_{k}+\sum_{\left(u_{j}, u_{k}\right) \in \operatorname{dit}(\pi)} p_{j} p_{k} .
\end{aligned}
$$

Thus the logical entropy with point probabilities is (using the point probability definition of $p\left(B_{i}\right)$ ):

$$
h_{p}(\pi)=\sum_{\left(u_{j}, u_{k}\right) \in \operatorname{dit}(\pi)} p_{j} p_{k}=\sum_{i \neq i^{\prime}} p\left(B_{i}\right) p\left(B_{i^{\prime}}\right)=2 \sum_{i<i^{\prime}} p\left(B_{i}\right) p\left(B_{i^{\prime}}\right)=1-\sum_{i=1}^{m} p\left(B_{i}\right)^{2} .
$$

One other version of the classical logical entropy might be mentioned. Instead of being given a partition $\pi=\left\{B_{1}, \ldots, B_{m}\right\}$ on $U$ with point probabilities $p_{j}$ defining the finite probability distribution of block probabilities $\left\{p\left(B_{i}\right)\right\}_{i}$, one might be given only a finite probability distribution $p=\left\{p_{1}, \ldots, p_{m}\right\}$. The substituting $p_{i}$ for $p\left(B_{i}\right)$ gives the:

$$
h(p)=1-\sum_{i=1}^{m} p_{i}^{2}
$$

logical entropy of a finite probability distribution.

There are also parallel element $\leftrightarrow$ distinction interpretations:

- $\operatorname{Pr}(S)=p_{S}$ is the probability that a single draw, sample, or experiment with $U$ gives a element $u_{j}$ of $S$, and

\footnotetext{
${ }^{2} \mathrm{~A}$ pair $\left\{i, i^{\prime}\right\}$ of distinct indices satisfies $i \neq i^{\prime}$ both ways so $\sum_{i \neq i^{\prime}} p_{B_{i}} p_{B_{i^{\prime}}}=2 \sum_{i<i^{\prime}} p_{B_{i}} p_{B_{i^{\prime}}}$.
} 
- $h_{p}(\pi)=\sum_{\left(u_{j}, u_{k}\right) \in \operatorname{dit}(\pi)} p_{j} p_{k}=\sum_{i \neq i^{\prime}} p\left(B_{i}\right) p\left(B_{i^{\prime}}\right)$ is the probability that two independent (with replacement) draws, samples, or experiments with $U$ gives a distinction $\left(u_{j}, u_{k}\right)$ of $\pi$, or if we interpret the independent experiments as sampling from the set of blocks $\pi=\left\{B_{i}\right\}$, then it is the probability of getting distinct blocks.

The parallelism or duality between logical probabilities and logical entropies is summarized in the following table.

\begin{tabular}{|l|l|l|}
\cline { 2 - 3 } \multicolumn{1}{c|}{} & Logical Probability Theory & Logical Information Theory \\
\hline 'Outcomes' & Elements $\mathrm{u} \in \mathrm{U}$ finite & Distinctions $\left(\mathrm{u}, \mathrm{u}^{\prime}\right) \in \mathrm{U} \times \mathrm{U}$ finite \\
\hline 'Events' & Subsets $\mathrm{S} \subseteq \mathrm{U}$ & $\operatorname{Dit~sets~} \operatorname{dit}(\pi) \subseteq \mathrm{U} \times \mathrm{U}$ \\
\hline $\begin{array}{l}\text { Equiprobable } \\
\text { outcomes }\end{array}$ & $\begin{array}{l}\operatorname{Pr}(\mathrm{S})=|\mathrm{S}| / \mathrm{U} \mid=\text { logical } \\
\text { probability of event } \mathrm{S}\end{array}$ & $\begin{array}{l}\mathrm{h}(\pi)=|\operatorname{dit}(\pi) / / \mathrm{U} \times \mathrm{U}|=\text { logical } \\
\text { entropy of partition } \pi\end{array}$ \\
\hline $\begin{array}{l}\text { Point } \\
\text { probabilities }\end{array}$ & $\begin{array}{l}\text { Pr}(\mathrm{S})=\Sigma\left\{\mathrm{p}_{\mathrm{j}}: \mathrm{u}_{\mathrm{j}} \in \mathrm{S}\right\}=\mathrm{p}(\mathrm{S})= \\
\text { logical prob. of event } \mathrm{S}\end{array}$ & $\begin{array}{l}\mathrm{h}_{\mathrm{p}}(\pi)=\Sigma\left\{\mathrm{p}_{\mathrm{j}} \mathrm{p}_{\mathrm{k}}:\left(\mathrm{u}_{\mathrm{j}}, \mathrm{u}_{\mathrm{k}}\right) \in \operatorname{dit}(\pi)\right\}= \\
\text { logical entropy of } \pi\end{array}$ \\
\hline Interpretation & $\begin{array}{l}\text { Pr}(\mathrm{S})=\text { one draw } \\
\text { probability of getting an } \\
\text { element from } \mathrm{S}\end{array}$ & $\begin{array}{l}\mathrm{h}_{\mathrm{p}}(\pi)=\text { two draw probability } \\
(\mathrm{w} / \text { replacement }) \text { of getting a } \\
\text { distinction of } \pi\end{array}$ \\
\hline
\end{tabular}

Figure 2: Classical logical probabilities and logical entropies.

\section{Classical logical entropy of a density matrix}

Before turning to the quantum case proper, we might consider a "classical" use of the notion of a density matrix [15] usually associated with quantum mechanics (QM). In the general case of a finite outcome set $U$ with point probabilities $p=\left\{p_{1}, \ldots, p_{n}\right\}$, then for any subset $S$ with $p(S)>0$ where $\chi_{S}\left(u_{i}\right)$ is the characteristic function for the subset $S$, we have a normalized column vector in $\mathbb{R}^{n}$ (with [ ${ }^{t}$ representing the transpose):

$$
|S\rangle=\frac{1}{\sqrt{p(S)}}\left[\chi_{S}\left(u_{1}\right) \sqrt{p_{1}}, \ldots, \chi_{S}\left(u_{n}\right) \sqrt{p_{n}}\right]^{t} .
$$

If we denote the corresponding row vector by $\langle S|$, then we may define the $n \times n$ density matrix $\rho(S)$ as:

$$
\frac{1}{p(S)}\left[\begin{array}{cccc}
\rho(S)=|S\rangle\langle S|= \\
\chi_{S}\left(u_{1}\right)^{2} p_{1} & \chi_{S}\left(u_{1}\right) \chi_{S}\left(u_{2}\right) \sqrt{p_{1} p_{2}} & \cdots & \chi_{S}\left(u_{1}\right) \chi_{S}\left(u_{n}\right) \sqrt{p_{1} p_{n}} \\
\chi_{S}\left(u_{2}\right) \chi_{S}\left(u_{1}\right) \sqrt{p_{2} p_{1}} & \chi_{S}\left(u_{2}\right)^{2} p_{2} & \cdots & \chi_{S}\left(u_{2}\right) \chi_{S}\left(u_{n}\right) \sqrt{p_{2} p_{n}} \\
\vdots & \vdots & \ddots & \vdots \\
\chi_{S}\left(u_{n}\right) \chi_{S}\left(u_{1}\right) \sqrt{p_{n} p_{1}} & \chi_{S}\left(u_{n}\right) \chi_{S}\left(u_{2}\right) \sqrt{p_{n} p_{2}} & \cdots & \chi_{S}\left(u_{n}\right)^{2} p_{n} \\
\text { Density matrix for a subset } S \subseteq U . &
\end{array}\right]
$$

It is then easy to characterize each entry in the matrix:

$$
(\rho(S))_{j k}=\left\{\begin{array}{c}
\frac{1}{p(S)} \sqrt{p_{j} p_{k}} \text { if } u_{j}, u_{k} \in S \\
0 \text { otherwise. }
\end{array}\right.
$$


Then a little calculation shows that the density matrix for a subset $S$ acts like a "pure state" in QM in the sense that it is idempotent $\rho(S)^{2}=\rho(S)$ since:

$$
\begin{gathered}
\left(\rho(S)^{2}\right)_{j k}=\frac{1}{p(S)^{2}} \sum_{l=1}^{n} \chi_{S}\left(u_{j}\right) \chi_{S}\left(u_{l}\right) \sqrt{p_{j} p_{l}} \chi_{S}\left(u_{l}\right) \chi_{S}\left(u_{k}\right) \sqrt{p_{l} p_{k}} \\
=\frac{1}{p(S)^{2}} \chi_{S}\left(u_{j}\right) \chi_{S}\left(u_{k}\right) \sqrt{p_{j} p_{k}} \sum_{l=1}^{n} \chi_{S}\left(u_{l}\right) p_{l}=\frac{1}{p(S)^{2}} \chi_{S}\left(u_{j}\right) \chi_{S}\left(u_{k}\right) \sqrt{p_{j} p_{k}} p(S)=(\rho(S))_{j k} .
\end{gathered}
$$

Then given a partition $\pi=\left\{B_{1}, \ldots, B_{m}\right\}$ on $U$, the density matrix for $\pi$, like a mixed-state density matrix in QM, is the probability-weighted sum of the density matrices for the blocks:

$$
\rho(\pi)=\sum_{i=1}^{m} p\left(B_{i}\right) \rho\left(B_{i}\right) .
$$

Density matrix for a partition $\pi$ on $U$.

Since $p\left(B_{i}\right) \times \frac{1}{p\left(B_{i}\right)} \sqrt{p_{j} p_{k}}=\sqrt{p_{j} p_{k}}$ for $\left(u_{j}, u_{k}\right) \in \operatorname{indit}(\pi)$, the entries in $\rho(\pi)$ are easily characterized:

$$
(\rho(\pi))_{j k}=\left\{\begin{array}{c}
\sqrt{p_{j} p_{k}} \text { if }\left(u_{j}, u_{k}\right) \in \operatorname{indit}(\pi) \\
0 \text { otherwise. }
\end{array}\right.
$$

Then the classical logical entropy formula $h_{p}(\pi)=1-\sum_{i=1}^{m} p\left(B_{i}\right)^{2}$ easily generalizes to density matrices by replacing the sum by the trace (sum of diagonal elements of a matrix) and the squared probabilities by the square of the density matrix:

$$
h(\rho(\pi))=1-\operatorname{tr}\left[\rho(\pi)^{2}\right]
$$

Logical entropy of a classical density matrix.

Since $\rho(S)^{2}=\rho(S)$ and the trace of any density matrix is 1, we immediately have $h(\rho(S))=0$, i.e., the logical entropy of a "pure state" subset $S \subseteq U$ is 0 .

We saw previously that $h_{p}(\pi)=1-\sum_{i=1}^{m} p\left(\bar{B}_{i}\right)^{2}$ so we need to check that the density matrix formulation gives the same result-which only requires a little calculation of the diagonal entries of $\rho(\pi)^{2}$. If $u_{j} \in B_{i}$, then

$$
\begin{aligned}
& \left(\rho(\pi)^{2}\right)_{j j}=\sum_{k=1}^{n} \rho(\pi)_{j k} \rho(\pi)_{k j} \\
= & p_{j} \sum_{k=1,\left(u_{j}, u_{k}\right) \in \operatorname{indit}(\pi)}^{n} p_{k}=p_{j} p\left(B_{i}\right)
\end{aligned}
$$

so the sum of the diagonal entries for $j$ where $u_{j} \in B_{i}$ is $p\left(B_{i}\right)^{2}$ and the sum of all the diagonal entries is:

$$
\operatorname{tr}\left[\rho(\pi)^{2}\right]=\sum_{i=1}^{m} p\left(B_{i}\right)^{2}=\sum_{\left(u_{j}, u_{k}\right) \in \operatorname{indit}(\pi)} p_{j} p_{k}
$$

Thus we have:

$$
h_{p}(\pi)=1-\sum_{i=1}^{m} p\left(B_{i}\right)^{2}=\sum_{\left(u_{j}, u_{k}\right) \in \operatorname{dit}(\pi)} p_{j} p_{k}=1-\operatorname{tr}\left[\rho(\pi)^{2}\right]=h(\rho(\pi)) .
$$

\section{Quantum logical entropy and measurement}

Let $V$ be an $n$-dimensional Hilbert space $\mathbb{C}^{n}$. If $\left|\psi_{i}\right\rangle$ for $i=1, \ldots, m$ is a set of normalized vectors from $V$ and $p=\left\{p_{1}, \ldots, p_{m}\right\}$ is a probability distribution, then

$$
\rho(\psi)=\sum_{i=1}^{m} p_{i}\left|\psi_{i}\right\rangle\left\langle\psi_{i}\right|
$$


is a density matrix, and any positive operator on $V$ of trace 1 has such an orthogonal decomposition $[15$, p. 101]. Then the quantum logical entropy $h(\rho)([7] ;[1])$ of a density matrix $\rho$ is defined as above for the classical logical entropy in terms of a "classical" density matrix:

$$
h(\rho)=1-\operatorname{tr}\left[\rho^{2}\right] .
$$

The formula $1-\operatorname{tr}\left[\rho^{2}\right]$ is not new in quantum information theory. ${ }^{3}$ Indeed, $\operatorname{tr}\left[\rho^{2}\right]$ is usually called the purity of the density matrix since a state $\rho$ is pure if and only if $\operatorname{tr}\left[\rho^{2}\right]=1$ so $h(\rho)=0$, and otherwise $\operatorname{tr}\left[\rho^{2}\right]<1$ so $h(\rho)>0$ and the state is said to be mixed. Hence the complement $1-\operatorname{tr}\left[\rho^{2}\right]$ has been called the "mixedness" [12, p. 5] of the state $\rho$. What is new is the whole backstory of partition logic outlined above which gives the logical notion of entropy arising out of partition logic as the normalized counting measure on partition relations-just as logical probability arises out of Boolean subset logic as the normalized counting measure on subsets. The basic idea of information is distinctions ([5], [9]), so the logical notion of entropy is just the (normalized) counting measure of distinctions of a partition and its various natural generalizations.

Quantum logical entropy $h(\rho)$ is to be compared to the Von Neumann entropy $S(\rho)=-\operatorname{tr}[\rho \log \rho]$ $[15$, p.510]. Along with Boaz and Cohen ([1], [2]), I would argue that the quantum logical entropy is a more natural and informative entropy concept for quantum mechanics than Von Neumann entropy.

We find this framework of partitions and distinction most suitable (at least conceptually) for describing the problems of quantum state discrimination, quantum cryptography and in general, for discussing quantum channel capacity. In these problems, we are basically interested in a distance measure between such sets of states, and this is exactly the kind of knowledge provided by logical entropy ([5]). [1, p. 1]

Our approach here is to compare what each notion of entropy tells about quantum measurement.

Let $F: V \rightarrow V$ be a self-adjoint operator with the real eigenvalues $\phi_{1}, \ldots, \phi_{m}$ and let $U=$ $\left\{u_{1}, \ldots, u_{n}\right\}$ an orthonormal basis of eigenvectors of $F$. Then there is a set partition $\pi=\left\{B_{i}\right\}_{i=1, \ldots, m}$ on $U$ so that $B_{i}$ is a basis for the eigenspace of the eigenvalue $\phi_{i}$ and $\left|B_{i}\right|$ is the "multiplicity" (dimension of the eigenspace) of the eigenvalue $\phi_{i}$ for $i=1, \ldots, m$. Note that the real-valued function $f: U \rightarrow \mathbb{R}$ that takes each eigenvector in $u_{j} \in B_{i} \subseteq U$ to its eigenvalue $\phi_{i}$ so that $f^{-1}\left(\phi_{i}\right)=B_{i}$ contains all the information in the self-adjoint operator $F: V \rightarrow V$ since $F$ can be reconstructed by defining it on the basis $U$ as $F u_{j}=f\left(u_{j}\right) u_{j}$.

In a measurement using a self-adjoint operator $F$, the operator does not provide the point probabilities; they come from the pure (normalized) state $\psi$ being measured. Let $|\psi\rangle=\sum_{j=1}^{n}\left\langle u_{j} \mid \psi\right\rangle\left|u_{j}\right\rangle=$ $\sum_{j=1}^{n} \alpha_{j}\left|u_{j}\right\rangle$ be the resolution of $|\psi\rangle$ in terms of the orthonormal basis $U=\left\{u_{1}, \ldots, u_{n}\right\}$ of eigenvectors for $F$. Then $p_{j}=\alpha_{j} \alpha_{j}^{*}\left(\alpha_{j}^{*}\right.$ is the complex conjugate of $\alpha_{j}$ ) for $j=1, \ldots, n$ are the point probabilities on $U$ and the pure state density matrix $\rho(\psi)=|\psi\rangle\langle\psi|$ has the entries: $\rho(\psi)_{j k}=\alpha_{j} \alpha_{k}^{*}$ so the diagonal entries $\rho(\psi)_{j j}=\alpha_{j} \alpha_{j}^{*}=p_{j}$ are the point probabilities. Let $S=\left\{u_{j} \in U: p_{j}>0\right\}$ be the support consisting of the points of positive probability.

One of our themes is the extent to which quantum calculations can be reformulated using classical set-based notions. The "classical" density matrix $\rho(S)$ defined above (using point probabilities) with $\rho(S)_{j k}=\frac{1}{p(S)} \sqrt{p_{j} p_{k}}=\sqrt{p_{j} p_{k}}\left(\right.$ since $p(S)=\sum_{p_{j}>0} p_{j}=1$ ) only has real entries while $\rho(\psi)=\alpha_{j} \alpha_{k}^{*}$ has complex entries, but they both have the same logical entropy of 0 :

$$
h(\rho(S))=1-\operatorname{tr}\left[\rho(S)^{2}\right]=0=1-\operatorname{tr}\left[\rho(\psi)^{2}\right]=h(\rho(\psi))
$$

since in both cases the density matrices are idempotent $\rho^{2}=\rho$ and $\operatorname{tr}[\rho]=1$.

Measurement turns pure states into mixed states. Let $\hat{P}_{i}$ for $i=1, \ldots, m$ be the projection operator to the eigenspace for $\phi_{i}$ so the $n \times n$ projection matrix in the basis $U$ is the diagonal matrix

\footnotetext{
${ }^{3}$ The classical logical entropy formula $1-\sum_{i} p_{i}^{2}$ as a measure of heterogeneity or diversity goes back to the early twentieth century $([5],[9])$.
} 
$P_{i}$ where $\left(P_{i}\right)_{j j}=1$ if $u_{j} \in B_{i}$ and otherwise 0, i.e., $\left(P_{i}\right)_{j j}=\chi_{B_{i}}\left(u_{j}\right)$, and let $\psi_{i}=\hat{P}_{i}(\psi)$. The probability that a (projective) measurement will have the outcome $\phi_{i}$ and thus project $\psi$ to $\psi_{i}$ is:

$$
p\left(B_{i}\right)=\sum_{u_{j} \in B_{i}} p_{j}=\sum_{u_{j} \in B_{j}} \alpha_{j} \alpha_{j}^{*}=\left\|\psi_{i}\right\|^{2}=\operatorname{tr}\left[P_{i} \rho(\psi)\right] .
$$

Normalizing $\left|\psi_{i}\right\rangle$ by its norm $\left\|\psi_{i}\right\|=\sqrt{p\left(B_{i}\right)}$ allows us to construct its density matrix as (where the conjugate transpose $P_{i}^{\dagger}=P_{i}$ ):

$$
\rho\left(\psi_{i}\right)=\frac{1}{\sqrt{p\left(B_{i}\right)}}\left|\psi_{i}\right\rangle\left\langle\psi_{i}\left|\frac{1}{\sqrt{p\left(B_{i}\right)}}=\frac{1}{p\left(B_{i}\right)} P_{i}\right| \psi\right\rangle\langle\psi| P_{i}^{\dagger}=\frac{1}{p\left(B_{i}\right)} P_{i} \rho(\psi) P_{i}
$$

The density matrix for the mixed state resulting from the projective measurement $[15$, p. 515$]$ is the probability weighted sum of projected density matrices $\rho\left(\psi_{i}\right)$ :

$$
\hat{\rho}(\psi)=\sum_{i=1}^{m} p\left(B_{i}\right) \rho\left(\psi_{i}\right)=\sum_{i=1}^{m} P_{i} \rho(\psi) P_{i} .
$$

Since the logical entropy of the pure state $\rho(\psi)$ was 0 , the increase in logical entropy resulting from the measurement is the logical entropy of $\hat{\rho}(\psi)$ :

$$
h(\hat{\rho}(\psi))=1-\operatorname{tr}\left[\hat{\rho}(\psi)^{2}\right] .
$$

In terms of sets, we have the set partition $\pi=\left\{B_{i}\right\}_{i=1, \ldots, m}$ on the set $U$ with the point probabilities $p=\left\{p_{j}\right\}_{j=1, \ldots, n}$ which has the classical logical entropy $h_{p}(\pi)=1-\sum_{i=1}^{m} p\left(B_{i}\right)^{2}$. As noted in the previous section, this can also be obtained as the logical entropy of the classical density matrix $\rho(\pi)$ of that partition:

$$
h_{p}(\pi)=1-\sum_{i=1}^{m} p\left(B_{i}\right)^{2}=1-\operatorname{tr}\left[\rho(\pi)^{2}\right]=h(\rho(\pi)) .
$$

Our first result is that the quantum logical entropy $h(\hat{\rho}(\psi))$ resulting from the projective measurement can be computed classically as $h_{p}(\pi)=h(\rho(\pi))$.

Proposition $1 h_{p}(\pi)=h(\hat{\rho}(\psi))$.

Proof: Pre- and post-multiplying $\rho(\psi)$ by the diagonal projection matrices $P_{i}$ with $\left(P_{i}\right)_{j j}=$ $\chi_{B_{i}}\left(u_{j}\right)$ gives:

$$
\left(P_{i} \rho(\psi) P_{i}\right)_{j k}=\left\{\begin{array}{c}
\alpha_{j} \alpha_{k}^{*} \text { if }\left(u_{j}, u_{k}\right) \in B_{i} \times B_{i} \\
0 \text { if not. }
\end{array}\right.
$$

and since indit $(\pi)=\cup_{i=1}^{m} B_{i} \times B_{i}$

$$
(\hat{\rho}(\psi))_{j k}=\left(\sum_{i=1}^{m} P_{i} \rho(\psi) P_{i}\right)_{j k}=\left\{\begin{array}{c}
\alpha_{j} \alpha_{k}^{*} \text { if }\left(u_{j}, u_{k}\right) \in \operatorname{indit}(\pi) \\
0 \text { if }\left(u_{j}, u_{k}\right) \in \operatorname{dit}(\pi)
\end{array} .\right.
$$

Hence to compute the quantum logical entropy $h(\hat{\rho}(\psi))$ :

$$
\left(\hat{\rho}(\psi)^{2}\right)_{j j}=\sum_{k} \hat{\rho}(\psi)_{j k} \hat{\rho}(\psi)_{k j}=\sum_{k,\left(u_{j}, u_{k}\right) \in \operatorname{indit}(\pi)}\left\|\alpha_{j}\right\|^{2}\left\|\alpha_{k}\right\|^{2}
$$

so the trace is:

$$
\begin{gathered}
\operatorname{tr}\left[\hat{\rho}(\psi)^{2}\right]=\sum_{j}\left(\hat{\rho}(\psi)^{2}\right)_{j j}=\sum_{j} \sum_{k,\left(u_{j}, u_{k}\right) \in \operatorname{indit}(\pi)}\left\|\alpha_{j}\right\|^{2}\left\|\alpha_{k}\right\|^{2} \\
=\sum_{\left(u_{j}, u_{k}\right) \in \operatorname{indit}(\pi)} p_{j} p_{k}
\end{gathered}
$$


and the quantum logical entropy is:

$$
\begin{aligned}
h(\hat{\rho}(\psi)) & =1-\operatorname{tr}\left[\hat{\rho}(\psi)^{2}\right]=1-\sum_{\left(u_{j}, u_{k}\right) \in \operatorname{indit}(\pi)} p_{j} p_{k} \\
& =\sum_{\left(u_{j}, u_{k}\right) \in \operatorname{dit}(\pi)} p_{j} p_{k}=h_{p}(\pi) .
\end{aligned}
$$

Corollary 1 The quantum logical entropy $h(\hat{\rho}(\psi))$ of the projective measurement result $\hat{\rho}(\psi)$ is the probability of getting different results $\left(\phi_{i} \neq \phi_{i^{\prime}}\right)$ in two independent measurements with the same observable and the same state.

The quantum logical entropy $h(\rho(\psi))$ and the Von Neumann entropy $S(\rho(\psi))$ for both zero for a pure state $\rho(\psi)^{2}=\rho(\psi)$, but the measurement entropies $h(\hat{\rho}(\psi))$ and $S(\hat{\rho}(\psi))$ are different, and the question is: "Which entropy concept gives insight into what happens in the quantum measurement?". The last corollary shows that the quantum logical entropy $h(\hat{\rho}(\psi))$ associated with the projective measurement has a very simple interpretation-whereas there seems to be no such simple interpretation for $S(\hat{\rho}(\psi))$. Our next and main result shows that the quantum logical entropy $h(\hat{\rho}(\psi)$ ) (which is also the increase in quantum logical entropy due to the projective measurement since $h(\rho(\psi))=0)$ is precisely related to what happens to the off-diagonal entries in the change in the density matrices $\rho(\psi) \rightarrow \hat{\rho}(\psi)$ due to the measurement-whereas no such specific result seems to hold for the Von Neumann entropy other than it also increases: $S(\rho(\psi))<S(\hat{\rho}(\psi))[15$, p. 515] $[$ when $\rho(\psi) \neq \hat{\rho}(\psi)]$.

Theorem 1 The quantum logical entropy $h(\hat{\rho}(\psi))$ is the sum of the absolute squares of the nonzero off-diagonal entries in the pure state density matrix $\rho(\psi)$ that are zeroed in the transition $\rho(\psi) \longrightarrow$ $\hat{\rho}(\psi)$ due to the projective measurement.

Proof: For $S=\left\{u_{j} \in U: p_{j}>0\right\}$, the nonzero elements of $\rho(\psi)$ are $(\rho(\psi))_{j k}=\alpha_{j} \alpha_{k}^{*}$ for $\left(u_{j}, u_{k}\right) \in S \times S$ with the absolute squares $\alpha_{j} \alpha_{k}^{*} \alpha_{j}^{*} \alpha_{k}=p_{j} p_{k}$. The post-measurement density matrix has the nonzero entries $(\hat{\rho}(\psi))_{j k}=\alpha_{j} \alpha_{k}^{*}$ if $\left(u_{j}, u_{k}\right) \in \operatorname{indit}(\pi) \cap S \times S$ where indit $(\pi)=\cup_{i=1}^{m} B_{i} \times B_{i}$. Hence the nonzero entries of $\rho(\psi)$ that got zeroed in $\hat{\rho}(\psi)$ are precisely the entries $(\rho(\psi))_{j k}=\alpha_{j} \alpha_{k}^{*}$ for $\left(u_{j}, u_{k}\right) \in \operatorname{dit}(\pi) \cap S \times S$. Since the entries for $\left(u_{j}, u_{k}\right) \notin S \times S$ were already zero, we have that the sum of the absolute squares of entries zeroed by the measurement is:

$$
\sum_{\left(u_{j}, u_{k}\right) \in \operatorname{dit}(\pi) \cap S \times S} p_{j} p_{k}=\sum_{\left(u_{j}, u_{k}\right) \in \operatorname{dit}(\pi)} p_{j} p_{k}=h(\hat{\rho}(\psi)) .
$$

In the classical version $\rho(S)$ of $\rho(\psi)$, the nonzero entries $\sqrt{p_{j} p_{k}}$ are the indistinction "amplitudes" whose square is the probability $p_{j} p_{k}$ of drawing $\left(u_{j}, u_{k}\right)$ in two independent draws from $S=\left\{u_{j} \in U: p_{j}>0\right\}$. In the equiprobable case of $p_{j}=\frac{1}{n}, S=U$ and the only partition with the classical logical entropy of zero is the indiscrete partition (or "blob") $\mathbf{0}=\{U\}$ which has no distinctions. But with point probabilities $\left\{p_{j}\right\}_{j}$ and $S \neq U$, the "outcomes" in $S^{c}=U-S$ have zero probability. The partition $\sigma=\left\{S, S^{c}\right\}$ has a nonempty $\operatorname{dit} \operatorname{set} \operatorname{dit}(\sigma)=\left(S \times S^{c}\right) \cup\left(S^{c} \times S\right)$ but clearly:

$$
h(\sigma)=\sum_{\left(u_{j}, u_{k}\right) \in \operatorname{dit}(\sigma)} p_{j} p_{k}=0=h(\rho(S))
$$

since $\sigma$ has no distinctions with positive probability-so $\sigma$ is effectively like the blob $\mathbf{0}$ as a classical "pure state."

The classical version of the measurement can be stated in classical finite probability theory where the outcome set or sample space $U=\left\{u_{1}, \ldots, u_{n}\right\}$ has point probabilities $p^{\prime}=\left\{p_{j}^{\prime}\right\}_{j=1, \ldots, n}$ for the outcomes. A real random variable $f: U \rightarrow \mathbb{R}$ has an image $f(U)=\left\{\phi_{1}, \ldots, \phi_{m}\right\}$ in the codomain $\mathbb{R}$ and induces a partition ("coimage") $\pi=\left\{f^{-1}\left(\phi_{i}\right)=B_{i}\right\}_{i=1, \ldots, m}$ on the domain $U$. Given an event or "state" $S \subseteq U$, the point probabilities for $u_{j} \in S$ can be conditionalized to $p_{j}=\frac{p_{j}^{\prime}}{\sum_{u_{k} \in S} p_{k}^{\prime}}=\frac{p_{j}^{\prime}}{p^{\prime}(S)}$ 
and set to 0 outside of $S$. The experiment to "measure" $f$ given the "state" $S$ returns a value $\phi_{i}$ with the probability $\operatorname{Pr}\left(\phi_{i} \mid S\right)=\sum_{u_{j} \in S \cap B_{i}} p_{j}=\frac{p^{\prime}\left(S \cap B_{i}\right)}{p^{\prime}(S)}$ and the "state" $S$ is reduced to the "state" $S \cap B_{i}$ with that probability. By "chopping up" the mini-blob $S$ into the partition $\left\{S \cap B_{i}\right\}_{i=1, \ldots, m}$ of $S$, the distinctions with nonzero probabilities in $\operatorname{dit}(\pi) \cap S \times S$ have been created and the sum of those distinction probabilities is the logical entropy: ${ }^{4}$

$$
\sum_{\left(u_{j}, u_{k}\right) \in \operatorname{dit}(\pi) \cap S \times S} p_{j} p_{k}=\sum_{\left(u_{j}, u_{k}\right) \in \operatorname{dit}(\pi)} p_{j} p_{k}=h_{p}(\pi) .
$$

Corollary 2 The classical logical entropy $h_{p}(\pi)=h(\rho(\pi))$, which equals $h(\hat{\rho}(\psi))$, is the sum of the squares of the nonzero off-diagonal entries in $\rho(S)$ that are zeroed in the transition $\rho(S) \rightarrow \rho(\pi)$.

Proof: The proof carries over substituting $\rho(S)$ for $\rho(\psi), \rho(\pi)$ for $\hat{\rho}(\psi)$, and $\sqrt{p_{j} p_{k}}$ for $\alpha_{j} \alpha_{k}^{*}$.

\section{Concluding remarks}

This main theorem about quantum logical entropy as well as the connection to classical logical entropy, i.e., information as distinctions, together with the backstory of partition logic allows some elucidation of a projective quantum measurement. The nonzero off-diagonal entries $\alpha_{j} \alpha_{k}^{*}$ in the density matrix $\rho(\psi)$ are called "coherences" [4, p. 303] because they give the amplitude of the eigenstates $\left|u_{j}\right\rangle$ and $\left|u_{k}\right\rangle$ "cohering" together in the coherent superposition state vector $|\psi\rangle=\sum_{j}\left\langle u_{j} \mid \psi\right\rangle\left|u_{j}\right\rangle=\sum_{j} \alpha_{j}\left|u_{j}\right\rangle$. The off-diagonal elements of $\rho(\psi)$ that are zeroed by the measurement to yield $\hat{\rho}(\psi)$ are the coherences (like "quantum indistinctions") that are turned into decoherences (like "quantum distinctions").

Measurement creates distinctions, i.e., turns indistinctions (or coherences) into distinctions (or decoherences), to make an indefinite state (superposition of definite eigenstates) more definite in the measured observable. The off-diagonal coherences in $\rho(\psi)$ are essentially the amplitudes for indistinctions so the ones that are zeroed are turned into distinctions (i.e., decoherences) and the sum of those distinction probabilities is the classical and quantum logical entropies.

\section{References}

[1] Tamir, Boaz, and Eliahu Cohen. 2014. Logical Entropy for Quantum States. ArXiv.org. December. http://de.arxiv.org/abs/1412.0616v2.

[2] Tamir, Boaz, and Eliahu Cohen. 2015. A Holevo-Type Bound for a Hilbert Schmidt Distance Measure. Journal of Quantum Information Science 5: 127-33.

[3] Boole, George 1854. An Investigation of the Laws of Thought on which are founded the Mathematical Theories of Logic and Probabilities. Cambridge: Macmillan and Co.

[4] Cohen-Tannoudji, Claude, Bernard Diu and Franck Laloë 2005. Quantum Mechanics Vol. 1. New York: John Wiley \& Sons.

[5] Ellerman, David. 2009. Counting Distinctions: On the Conceptual Foundations of Shannon's Information Theory. Synthese 168 (1 May): 119-49.

\footnotetext{
${ }^{4}$ This description of a classical version of quantum measurement suggests a pedagogical model of finite dimensional QM where the probability calculus is classical finite probability theory (with equiprobable outcomes or point probabilities). That model can be formulated in the vector space $\mathbb{Z}_{2}^{n}$ to allow for different and incompatible bases $U^{\prime}$ of $\mathbb{Z}_{2}^{n}$ so the resulting "quantum mechanics over sets" or QM/Sets has a non-commutative version of classical finite probability theory as its probability calculus.([8], or more rigorously, [11])
} 
[6] Ellerman, David 2010. The Logic of Partitions: Introduction to the Dual of the Logic of Subsets. Review of Symbolic Logic. 3 (2 June): 287-350.

[7] Ellerman, David. 2013. The Objective Indefiniteness Interpretation of Quantum Mechanics. ArXiv.org. January. http://arxiv.org/abs/1210.7659v2.

[8] Ellerman, David 2013. Quantum mechanics over sets. arXiv:1310.8221 [quant-ph].

[9] Ellerman, David. 2013. An Introduction to Logical Entropy and Its Relation to Shannon Entropy. International Journal of Semantic Computing 7 (2): 121-45.

[10] Ellerman, David 2014. An Introduction of Partition Logic. Logic Journal of the IGPL. 22, no. 1: $94-125$.

[11] Ellerman, David. 2016. The Quantum Logic of Direct-Sum Decompositions. ArXiv.org. April. http://arxiv.org/abs/1604.01087v2.

[12] Jaeger, Gregg. 2007. Quantum Information: An Overview. New York: Springer Science+Business Media.

[13] Laplace, Pierre-Simon. 1995 (1825). Philosophical Essay on Probabilities. Translated by A.I. Dale. New York: Springer Verlag.

[14] Lawvere, F. William and Robert Rosebrugh 2003. Sets for Mathematics. Cambridge: Cambridge University Press.

[15] Nielsen, M., and I. Chuang. 2000. Quantum Computation and Quantum Information. Cambridge: Cambridge University Press. 